\title{
An Efficient Biological Sequence Compression Technique Using LUT and Repeat in the Sequence
}

\author{
Subhankar Roy ${ }^{1}$, Sunirmal Khatua ${ }^{2}$, Sudipta Roy ${ }^{3}$, Prof. Samir K. \\ Bandyopadhyay $^{4}$ \\ ${ }^{1}$ Department of Computer Science \& Engineering, GNIT, WBUT, Kolkata, India \\ ${ }^{2,3,4}$ Department of Computer Science and Engineer, University of Calcutta, \\ 92 A.P.C. Road, Kolkata-700009, India
}

\begin{abstract}
Data compression plays an important role to deal with high volumes of DNA sequences in the field of Bioinformatics. Again data compression techniques directly affect the alignment of DNA sequences. So the time needed to decompress a compressed sequence has to be given equal priorities as with compression ratio. This article contains first introduction then a brief review of different biological sequence compression after that my proposed work then our two improved Biological sequence compression algorithms after that result followed by conclusion and discussion, future scope and finally references. These algorithms gain a very good compression factor with higher saving percentage and less time for compression and decompression than the previous Biological Sequence compression algorithms.
\end{abstract}

Keywords: Hash map table, Tandem repeats, compression factor, compression time, saving percentage, compression, decompression process.

\section{Introduction}

Compression arises because approximately $139,266,481,398$ bases (139.3 billion) from 151,824,421(153 million) reported sequences are there in the GenBank database in April 15, 2012[1] and the database size getting two or three times bigger annually. Compression required so that large data sequence after compression can easily be transferred through network channel to analyst. Data compression decrease communication cost by speed up transmission due to limited bandwidth communication of modern world and storage cost. This is very useful when processing, storing or transferring a huge file, which needs lots of resources. It is a well known fact that biological sequence compression is a useful tool to recover information from biological sequences. It has also been observed that better compression gives better understanding as it is sent to different station for its observation. Hence, compression of Biological sequences is a need for analyst for its storage, alignment and analyzing from remote locations and indeed a very challenging task. The Biological sequence consist of four nucleotide bases $\{\mathrm{A}, \mathrm{C}, \mathrm{G}, \mathrm{T}\}$, so only two bits are enough to store each base. Sometimes a special character $\mathrm{N}$ appears in some sequence which have equal probability of being A, C, G and T but it is an exceptional case. Other character like R, Y, W, S, M, K, H, B, V and D may appear in some sequence but they are very rare cases.

General text compression algorithms do not utilize the specific characteristics of DNA sequences i.e. more redundant nature of Biological sequences. The standard techniques of text compression do not compress these sequences; rather they expand the size of file with more than 2 bits per bases. Even though these compression tools are universal compression algorithms are designed for text compression. The regularities in Biological sequences are more.

It is known that the compression means mapping between source file and destination file, so our compression algorithm leads to find those relationship.

The objective of this paper is to build a finite LUT in which the combination of three and four characters of characters $\mathrm{A}, \mathrm{C}, \mathrm{G}, \mathrm{T}$ and $\mathrm{N}$ are taken as input and by mapping relationship during compression process output correspond to ASCII character and then find tandem repeats in the sequence for the first case. We compress the individual DNA sequences, not the whole genome sequence [2].

\section{Brief review}

Several compression algorithms specialized for Biological sequences have been developed in the last decade and some of these are; DNABIT compress tool (DBC) [3], GENBIT compress tool (GBC) [4], HUFFBIT COMPRESS [5] etc. One knows that all such algorithms take a long time but achieving high speed and best compression factor remains to be a challenging task. In this article, it has been tried to cope the above said problem. In this work it has been tried to achieve a better compression factor, saving percentage and runs significantly faster than existing compression program. 
Research work has been carried out for developing efficient Biological sequence compression technique. Some of them use the property similarity of genomic sequence [6], approximate matching [7], complementary palindrome [8] and cross chromosomal similarities within sequences [9] etc. In present work the properties tandem repeat in the sequences has been utilized.

\section{Proposed work}

First step of coding rule is based on LUT.

For algorithm-1, we take all possible three characters combination of characters $\{\mathrm{A}, \mathrm{T}, \mathrm{C}, \mathrm{G}$ or $\mathrm{N}\}$, so total $5^{\wedge} 3=125$ combinations are formed. Then we mapped those 125 combinations into 125 chosen ASCII character 8 bit each. The generated LUT is given in table 1.Here it has been also observed that any sequence file of characters a, t, $\mathrm{g}, \mathrm{c}$ or $\mathrm{n}$ and $\mathrm{A}, \mathrm{T}, \mathrm{G}, \mathrm{C}$ or $\mathrm{N}$ have same meaning. As an example if a line segment

Table 1: Look up table (map 3 to 1)

\begin{tabular}{|c|c|c|c|c|c|c|c|c|c|}
\hline Base & Character & Base & Character & Base & Character & Base & Character & Base & Character \\
\hline AAA & B & TAA & - & CAA & \} & GAA & 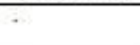 & NAA & $\Phi$ \\
\hline AAT & D & TAT & & CAT & $\sim$ & GAT & , & NAT & $\ddot{\mathrm{N}}$ \\
\hline AAC & $E$ & TAC & $\mathrm{b}$ & CAC & 0 & GAC & 1 & NAC & 0 \\
\hline$\overline{\mathrm{AAG}}$ & F & TAG & $\mathrm{d}$ & CAG & $i$ & GAG & . & NAG & 0 \\
\hline$\overline{\mathrm{AAN}}$ & $\mathrm{H}$ & TAN & $\mathrm{e}$ & CAN & $c$ & GAN & ") & NAN & 0 \\
\hline ATA & I & TTA & $f$ & CTA & $£$ & GTA & $1 / 4$ & NTA & $\dot{0}$ \\
\hline ATT & $\mathrm{J}$ & TTT & $\mathrm{h}$ & CTT & 0 & GTT & $1 / 2$ & NTT & $\ddot{0}$ \\
\hline ATC & $\mathrm{K}$ & TTC & $\mathrm{i}$ & CTC & $¥$ & GTC & $3 / 4$ & NTC & $x$ \\
\hline ATG & L & TTG & j & CTG & 1 & GTG & $i$ & NTG & $\varnothing$ \\
\hline$\overline{A T N}$ & M & TTN & $\mathrm{k}$ & CTN & $\S$ & GTN & A & NTN & $\mathrm{U}$ \\
\hline$\overline{\mathrm{ACA}}$ & 0 & TCA & 1 & $\mathrm{CCA}$ & 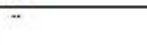 & GCA & $\mathrm{A}$ & NCA & $\mathrm{U}$ \\
\hline ACT & $P$ & TCT & $\mathrm{m}$ & CCT & ( ) & GCT & $\overline{\mathrm{A}}$ & NCT & $\bar{U}$ \\
\hline$\overline{\mathrm{ACC}}$ & $Q$ & TCC & 0 & $\mathrm{CCC}$ & $a$ & GCC & $\dot{A}$ & $\mathrm{NCC}$ & $\overline{\mathrm{U}}$ \\
\hline ACG & $\mathrm{R}$ & TCG & $p$ & $\mathrm{CCG}$ & " & $\mathrm{GCG}$ & $\ddot{A}$ & $\mathrm{NCG}$ & $\mathrm{Y}$ \\
\hline$\overline{\mathrm{ACN}}$ & $\mathrm{S}$ & TCN & $q$ & $\mathrm{CCN}$ & - & $\mathrm{GCN}$ & $\bar{\AA}$ & $\mathrm{NCN}$ & p \\
\hline$\overline{\text { AGA }}$ & U & TGA & $\mathrm{r}$ & CGA & - & GGA & E & NGA & B \\
\hline$\overline{\text { AGT }}$ & V & TGT & $s$ & CGT & (8) & GGT & Ç & NGT & $\bar{a}$ \\
\hline$\overline{\text { AGC }}$ & W & TGC & $\mathrm{u}$ & CGC & & GGC & $E$ & NGC & $\dot{a}$ \\
\hline$\overline{\mathrm{AGG}}$ & $\mathrm{X}$ & TGG & $\mathrm{v}$ & CGG & $\circ$ & GGG & $E$ & NGG & $\bar{a}$ \\
\hline AGN & $\mathrm{Y}$ & TGN & $\mathrm{w}$ & CGN & \pm & GGN & $\bar{E}$ & $\mathrm{NGN}$ & $\tilde{a}$ \\
\hline ANA & Z & TNA & $\mathrm{x}$ & CNA & $2^{2}$ & GNA & $\ddot{\bar{E}}$ & NNA & $\ddot{a}$ \\
\hline ANT & [ & TNT & $y$ & CNT & 3 & GNT & I & NNT & $\bar{a}$ \\
\hline ANC & 1 & TNC & $z$ & CNC & & GNC & I & NNC & 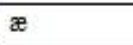 \\
\hline ANG & ] & TNG & \{ & CNG & $\mu$ & GNG & I & NNG & ६ \\
\hline ANN & $\wedge$ & TNN & $T$ & $\mathrm{CNN}$ & T & GNN & $\overline{\mathrm{I}}$ & NNN & $\overline{\mathrm{e}}$ \\
\hline
\end{tabular}

"AAANNNTTTGGGCCC" appeared in the input text, in the output file, it is represented as "BèhÉ"." The generated output is become case-sensitive.

For algorithm-2, we take all possible four characters combination of characters $\{\mathrm{A}, \mathrm{T}, \mathrm{C}$ and $\mathrm{G}\}$, so total $4^{\wedge} 4=256$ combinations are formed. Then we mapped those 256 combinations into 256 ASCII character 8 bit each. We have not taken into account character ' $\mathrm{N}$ ', because this is an exceptional case. The generated LUT is shown in table 2.For example for line segment "CAAACTTACTGA" output is " $E$ " $œ "$. 


\begin{tabular}{|c|c|c|c|c|c|c|c|}
\hline \multicolumn{8}{|c|}{ Table 2: Look up table (map 4 to 1) } \\
\hline Base & Character & Base & Character & Base & Character & Base & Character \\
\hline AAAA & (char)0 & TAAA & (char)64 & CAAA & (char)128 & GAAA & (char)192 \\
\hline AAAT & (char) 1 & TAAT & (char)65 & CAAT & (char)129 & GAAT & (char)193 \\
\hline AAAC & (char)2 & TAAC & (char)66 & CAAC & (char)130 & GAAC & (char)194 \\
\hline$\overline{\mathrm{AAAG}}$ & (char)3 & TAAG & (char)67 & CAAG & (char)131 & GAAG & (char)195 \\
\hline AATA & (char)4 & TATA & (char)68 & CATA & (char)132 & GATA & (char)196 \\
\hline AATT & (char)5 & TATT & (char)69 & CATT & (char)133 & GATT & (char)197 \\
\hline AATC & (char)6 & TATC & (char)70 & CATC & (char)134 & GATC & (char)198 \\
\hline AATG & (char)7 & TATG & (char)71 & CATG & (char)135 & GATG & (char)199 \\
\hline AACA & (char)8 & TACA & (char)72 & CACA & (char)136 & GACA & (char)200 \\
\hline AACT & (char)9 & TACT & (char)73 & CACT & (char)137 & GACT & (char)201 \\
\hline AACC & (char)10 & TACC & (char)74 & CACC & (char)138 & GACC & (char)202 \\
\hline AACG & (char)11 & TACG & (char)75 & CACG & (char)139 & GACG & (char)203 \\
\hline AAGA & (char)12 & TAGA & (char)76 & CAGA & (char)140 & GAGA & (char)204 \\
\hline AAGT & (char)13 & TAGT & (char)77 & CAGT & (char)141 & GAGT & (char)205 \\
\hline AAGC & (char)14 & TAGC & (char)78 & CAGC & (char)142 & GAGC & (char)206 \\
\hline$\overline{\mathrm{AAGG}}$ & (char)15 & TAGG & (char)79 & CAGG & (char)143 & GAGG & (char)207 \\
\hline ATAA & (char)16 & TTAA & (char) 80 & CTAA & (char)144 & GTAA & (char)208 \\
\hline ATAT & (char)17 & TTAT & (char)81 & $\begin{array}{l}\text { CTAT } \\
\end{array}$ & (char)145 & GTAT & (char)209 \\
\hline ATAC & 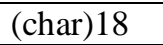 & TTAC & (char)82 & CTAC & (char)146 & GTAC & (char)210 \\
\hline ATAG & (char)19 & TTAG & (char)83 & CTAG & (char)147 & GTAG & (char)211 \\
\hline ATTA & (char)20 & TTTA & (char)84 & CTTA & (char)148 & GTTA & (char)212 \\
\hline ATTT & (char)21 & TTTT & (char)85 & CTTT & (char)149 & GTTT & (char)213 \\
\hline ATTC & (char)22 & TTTC & (char)86 & CTTC & (char)150 & GTTC & (char)214 \\
\hline ATTG & (char)23 & TTTG & (char)87 & CTTG & (char)151 & GTTG & (char)215 \\
\hline ATCA & (char)24 & TTCA & (char)88 & CTCA & (char)152 & GTCA & (char)216 \\
\hline ATCT & (char)25 & TTCT & (char)89 & CTCT & (char)153 & GTCT & (char)217 \\
\hline ATCC & (char)26 & TTCC & (char)90 & CTCC & (char)154 & GTCC & (char)218 \\
\hline ATCG & (char)27 & TTCG & (char)91 & CTCG & (char)155 & GTCG & (char)219 \\
\hline ATGA & (char)28 & TTGA & (char)92 & CTGA & (char)156 & GTGA & (char)220 \\
\hline ATGT & (char)29 & TTGT & (char)93 & CTGT & (char)157 & GTGT & (char)221 \\
\hline ATGC & (char)30 & TTGC & (char)94 & CTGC & (char)158 & GTGC & (char)222 \\
\hline ATGG & (char)31 & TTGG & (char)95 & CTGG & (char)159 & GTGG & (char)223 \\
\hline ACAA & (char)32 & TCAA & (char)96 & CCAA & (char)160 & GCAA & (char)224 \\
\hline ACAT & (char) 33 & TCAT & (char)97 & CCAT & (char)161 & GCAT & (char)225 \\
\hline ACAC & (char)34 & TCAC & (char)98 & CCAC & (char)162 & GCAC & (char)226 \\
\hline ACAG & (char)35 & TCAG & (char)99 & CCAG & (char)163 & GCAG & (char)227 \\
\hline ACTA & (char)36 & TCTA & (char)100 & CCTA & (char)164 & GCTA & (char)228 \\
\hline ACTT & (char)37 & TCTT & (char)101 & CCTT & (char)165 & GCTT & (char)229 \\
\hline ACTC & (char)38 & TCTC & (char)102 & CCTC & (char)166 & GCTC & (char)230 \\
\hline ACTG & (char)39 & TCTG & (char)103 & CCTG & (char)167 & GCTG & (char)231 \\
\hline ACCA & (char)40 & TCCA & (char)104 & CCCA & (char)168 & GCCA & (char)232 \\
\hline ACCT & (char)41 & TCCT & (char)105 & CCCT & (char)169 & GCCT & (char)233 \\
\hline ACCC & (char)42 & TCCC & (char)106 & CCCC & (char)170 & GCCC & (char)234 \\
\hline ACCG & (char)43 & TCCG & (char)107 & CCCG & (char)171 & GCCG & (char)235 \\
\hline ACGA & (char)44 & TCGA & (char)108 & CCGA & (char) 172 & GCGA & (char)236 \\
\hline ACGT & (char)45 & TCGT & (char)109 & CCGT & (char)173 & GCGT & (char)237 \\
\hline ACGC & (char)46 & TCGC & (char)110 & CCGC & (char)174 & GCGC & (char)238 \\
\hline ACGG & (char)47 & TCGG & (char)111 & CCGG & (char) 175 & GCGG & (char)239 \\
\hline AGAA & (char)48 & TGAA & (char)112 & CGAA & (char)176 & GGAA & (char)240 \\
\hline AGAT & (char)49 & TGAT & (char)113 & CGAT & (char)177 & GGAT & (char)241 \\
\hline AGAC & (char) 50 & TGAC & (char)114 & CGAC & (char)178 & GGAC & (char)242 \\
\hline AGAG & (char)51 & TGAG & (char)115 & CGAG & (char)179 & GGAG & (char)243 \\
\hline AGTA & (char)52 & TGTA & (char)116 & CGTA & (char)180 & GGTA & (char)244 \\
\hline AGTT & (char)53 & TGTT & (char)117 & CGTT & (char) 181 & GGTT & (char)245 \\
\hline AGTC & (char)54 & TGTC & (char)118 & CGTC & (char)182 & GGTC & (char)246 \\
\hline
\end{tabular}




\begin{tabular}{|l|l|l|l|l|l|l|l|}
\hline AGTG & (char)55 & TGTG & (char)119 & CGTG & (char)183 & GGTG & (char)247 \\
\hline AGCA & (char)56 & TGCA & (char)120 & CGCA & (char)184 & GGCA & (char)248 \\
\hline AGCT & (char)57 & TGCT & (char)121 & CGCT & (char)185 & GGCT & (char)249 \\
\hline AGCC & (char)58 & TGCC & (char)122 & CGCC & (char)186 & GGCC & (char)250 \\
\hline AGCG & (char)59 & TGCG & (char)123 & CGCG & (char)187 & GGCG & (char)251 \\
\hline AGGA & (char)60 & TGGA & (char)124 & CGGA & (char)188 & GGGA & (char)252 \\
\hline AGGT & (char)61 & TGGT & (char)125 & CGGT & (char)189 & GGGT & (char)253 \\
\hline AGGC & (char)62 & TGGC & (char)126 & CGGC & (char)190 & GGGC & (char)254 \\
\hline AGGG & (char)63 & TGGG & (char)127 & CGGG & (char)191 & GGGG & (char)255 \\
\hline
\end{tabular}

Second step of coding rule is based on ASCII character.

In algorithm-1 as the total number of ASCII required 125 so we can choose ASCII value out of total 256 ASCII value. That is why in this algorithm no problem occurred during the hash mapping.

But in algorithm-2 as all the ASCII value needed some problem arise during the mapping. The problem is that ASCII character of ASCII value 10 and 13 giving new line, as we compress and decompress file line by line so if we put those character in LUT during writing to file by hash mapping line break occurs. So we write the original characters block corresponding to these two values to the compress file. Another problem is five extended ASCII value (129, 141, 143, 144 and 157) not giving the correct characters so they cannot be map. So during compression if those ASCII value appear we do not map them using hash map table rather than we write the original block of characters value. That is why during decompression we have to take into account those characters block i.e. we have to search for those characters block because for them no reverse mapping is required.

Third step of coding rule is based on tandem repeats.

For algorithm-1 this feature can be used because during compression we count the number of repeats and write the corresponding character value to the compressed file. It has been observed that maximum number of repeats block never exceed 65 i.e. 195 character for any of the used sequences. So value 1 to 65 is used for handling those repeats except value 10 and 13 cause giving above. The repeat character followed by the number of repeats is written to the file. For e.g. input "AATAATAATAATAATAAC" have 4 tandem repeats. So these sequence represented by total 3 characters.

For algorithm-2 this feature cannot used because all ASCII value already used during mapping.

Fourth step of coding rule is based on Segment which consists of 1 or 2 characters.

We compress the input sequence line by line both for algorithm-1 and algorithm-2. For first algorithm we divide each line of the input sequence into segment of length 3 so if we modulate the line length by 3 i.e. Line length $\% 3=2$ or 1 or 0 . So the segments of 2 or 1 character are written as it is to the output file in the same line i.e. no mapping.

For second algorithm we divide each line of the input sequence into segment of length 4 so if we modulate the line length by 4 i.e. Line length $\% 4=3$ or 2 or 1 or 0 . So the segments of 3 or 2 or 1 character are written as it is to the output file no mapping but after a line break because they cannot be identify as 256 ASCII value already being used during mapping.

Algorithm for block size 3 with tandem repeats is given below

\subsection{Algorithm-1}

\subsubsection{Compression algorithm}

Input : Text file contain characters $\mathrm{A}, \mathrm{C}, \mathrm{G}$ and $\mathrm{T}$ or $\mathrm{a}, \mathrm{c}, \mathrm{g}$ and $\mathrm{t}$ or $\mathrm{A}, \mathrm{C}, \mathrm{G}, \mathrm{T}$ and $\mathrm{N}$ or $\mathrm{a}, \mathrm{c}, \mathrm{g}, \mathrm{t}$ and $\mathrm{n}$ respectively. Output: Compressed file in terms of ASCII characters.

Function Compression (Original file)

Step1: An ArrayList is made of all possible input characters.

Step2: A HashMap table map string to character is made dynamically at the run time of compression.

Step3: While string $<>$ null do //string reads the content of the file line by line

While string.length $>3$ do

Read a combination of three characters block and add them in a list.

End while

Add the last block to the same list.

For String string 1: list do

If string1.length $=3$ then

If string $1=$ temp then $/ /$ where temp store previous three character, initially null

count $=$ count $+1 / /$ count the number of repeats

Else \{

If count $>0$ then 


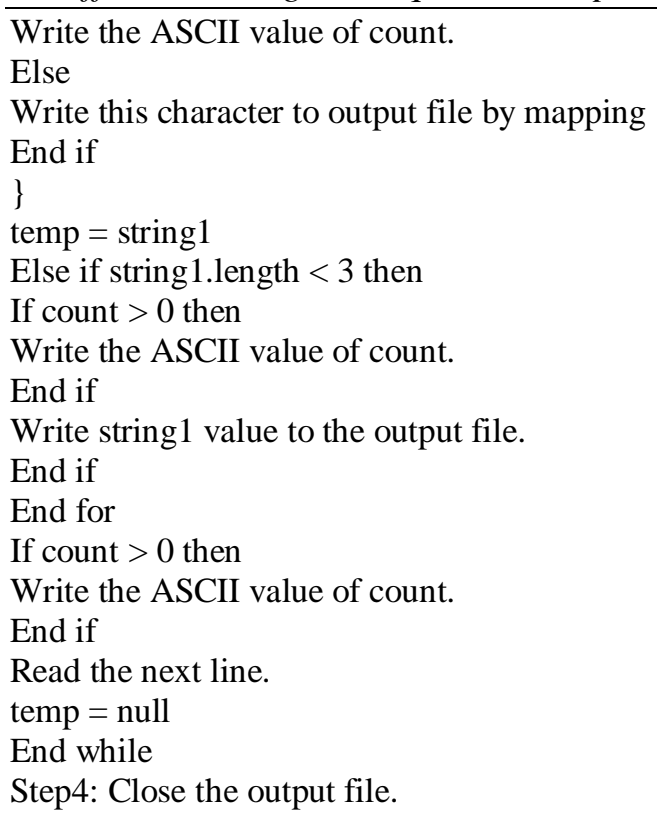

\subsubsection{Decompression algorithm}

Input : Compressed file.

Output : Original file.

Function Decompression (Compressed file)

Step1: An ArrayList is made of all possible input characters.

Step2: A HashMap table is made dynamically at the run time of compression.

Step3: Read the content of text file

While string <>null do

For 1 to string.length in step 1 do

Read a character from the string

If character $=$ ' $A$ ' or ' $a$ ' or ' $C$ ' or 'c' or ' $G$ ' or ' $g$ ' or ' $T$ ' or ' $t$ ' or ' $N$ ' or ' $n$ ' then

Write character to the output file.

Else \{

If count $>=1$ and $<=65$ then

For 1 to count in step 1 do

Write string of 3 characters to the file.

End for

Else

Write string of 3 characters to the file.

End if

\}

End if

End for

Read the next line

End while

Step4: Close the output file.

Algorithm for block size 4 without tandem repeats is given below

\subsection{Algorithm-2}

\subsubsection{Compression algorithm}

Input : Text file contains character $\mathrm{A}, \mathrm{C}, \mathrm{G}$ and $\mathrm{T}$ or a,c,g and t respectively.

Output : Compressed file in terms of all ASCII characters.

Function Compression (Text file)

Step1: An ArrayList is made of all possible input characters.

Step2: A HashMap table map string to character is made dynamically at the run time of compression.

Step3: Read the content of file.

While string <> null do

temp $=$ string $/ /$ temp store each line temporarily

While string.length ()$>4$ do 
Read a combination of four characters block.

Add them in a list.

End while

Add the last block to the same list.

For String string1: list do

If string1.length ()$=4$ then

Read the mapped character of block.

If $\mathrm{i}=10$ or 13 or 129 or 141 or 143 or 144 or 157 then // i store the integer value of the character.

Write the original block.

Else

Write the character.

End if

Else \{

Write newline character followed by block.

\}

End if

End for

Read the next line.

End while

Step4: Close the output file.

\subsubsection{Decompression algorithm}

Input : Compressed file.

Output : Original file.

Function Decompression (Compressed file)

Step1: An ArrayList is made of all possible input characters.

Step2: A HashMap table map string to character is made dynamically at the run time of compression.

Step3: Read the content of file.

While string $\langle>$ null do

If (count mod $2<>0$ ) then //Initially count=1

A HashMap table, hMap, map integer to string value.

A List intList of integer initially set to null.

Add block corresponding to 10,13, 129, 141, 143, 144 and 157 to an ArrayList strList.

For String str : strList do

intList := findIndexes(string, str)

For int index : intList do

hMap.put (index, str)

End for

End for

A Set keys of integer store the key value of hMap.

An ArrayList L of integer addAll the keys.

A Collection sort the value in $\mathrm{L}$.

For int midIndex : L do

For startIndex to midIndex in step 1 do $/ /$ startIndex $=0$

Read c character from string.

Write the corresponding string value by mapping to file.

End for

Write string value to file of midIndex by mapping.

StartIndex := midIndex +4 ;

End for

For startIndex to string.length() in step 1 do //Read remaining character

Read c character from string.

Write the corresponding string value by mapping to file.

End for

count $:=$ count +1 ;

Else \{

Write the same characters to file.

count $:=$ count +1 ;

Write newline character.

\} 
Read the next line.

End while

Step4: Close the output file.

\section{Results}

We have used three Homo sapiens mRNA: IL4-transcript variant 1, IL4-transcript variant 2 and MOPT, five funguses Rhizopus oryzae glucoamylase A precursor, gene, partial cds: strain 7, strain 15, strain 30, strain 45 and strain 52 that lives worldwide in dead organic matter and four Zea mays mRNA: ZM_BFb0129K09, ZM_BFb0203I02, ZM_BFc0025I23 and ZM_BFc0038A03 known corn or mielie/mealie, is a grain.

Table 3: Comparison of Compress Sequence Length (bytes) for different techniques

\begin{tabular}{|l|l|l|l|l|l|}
\hline Sequence Name & $\begin{array}{l}\text { Original } \\
\text { Seq. } \\
\text { Length } \\
\text { IMPLOD } \\
\text { E +LWZ }\end{array}$ & $\begin{array}{l}\text { Deflate + } \\
\text { PPM }\end{array}$ & $\begin{array}{l}\text { Block3 } \\
+ \\
\text { Tandem } \\
\text { repeats }\end{array}$ & Block4 \\
\hline IL4, transcript variant 1 & 660 & 402 & 358 & 234 & 225 \\
\hline IL4, transcript variant 2 & 610 & 379 & 333 & 214 & 208 \\
\hline MOPT & 712 & 377 & 355 & 254 & 241 \\
\hline strain 7 & 591 & 341 & 314 & 209 & 192 \\
\hline strain 15 & 589 & 344 & 316 & 210 & 199 \\
\hline strain 30 & 445 & 294 & 264 & 156 & 150 \\
\hline strain 45 & 594 & 341 & 311 & 210 & 201 \\
\hline strain 52 & 542 & 334 & 301 & 192 & 180 \\
\hline ZM_BFb0129K09 & 682 & 385 & 353 & 244 & 231 \\
\hline ZM_BFb0203I02 & 787 & 425 & 391 & 282 & 266 \\
\hline ZM_BFc0025I23 & 811 & 417 & 390 & 290 & 262 \\
\hline ZM_BFc0038A03 & 765 & 412 & 381 & 266 & 262 \\
\hline
\end{tabular}

Table 4: Comparison of Compression Factor for different techniques

\begin{tabular}{|l|l|l|l|l|}
\hline Sequence Name & $\begin{array}{l}\text { Deflate + } \\
\text { IMPLOD } \\
\text { E+LWZ }\end{array}$ & $\begin{array}{l}\text { LZSS+ } \\
\text { PPM }\end{array}$ & $\begin{array}{l}\text { Block3 } \\
+ \\
\text { Tandem } \\
\text { repeats }\end{array}$ & Block4 \\
\hline IL4, transcript variant 1 & 1.642 & 1.843 & 2.821 & 2.933 \\
\hline IL4, transcript variant 2 & 1.609 & 1.832 & 2.851 & 2.933 \\
\hline MOPT & 1.889 & 2.005 & 2.803 & 2.954 \\
\hline strain 7 & 1.733 & 1.882 & 2.827 & 3.078 \\
\hline strain 15 & 1.712 & 1.864 & 2.804 & 2.960 \\
\hline strain 30 & 1.514 & 1.686 & 2.853 & 2.967 \\
\hline strain 45 & 1.742 & 1.909 & 2.829 & 2.955 \\
\hline strain 52 & 1.623 & 1.801 & 2.823 & 3.011 \\
\hline ZM_BFb0129K09 & 1.771 & 1.932 & 2.795 & 2.952 \\
\hline ZM_BFb0203I02 & 1.852 & 2.013 & 2.791 & 2.959 \\
\hline ZM_BFc0025I23 & 1.945 & 2.079 & 2.797 & 3.095 \\
\hline ZM_BFc0038A03 & 1.857 & 2.008 & 2.876 & 2.921 \\
\hline
\end{tabular}




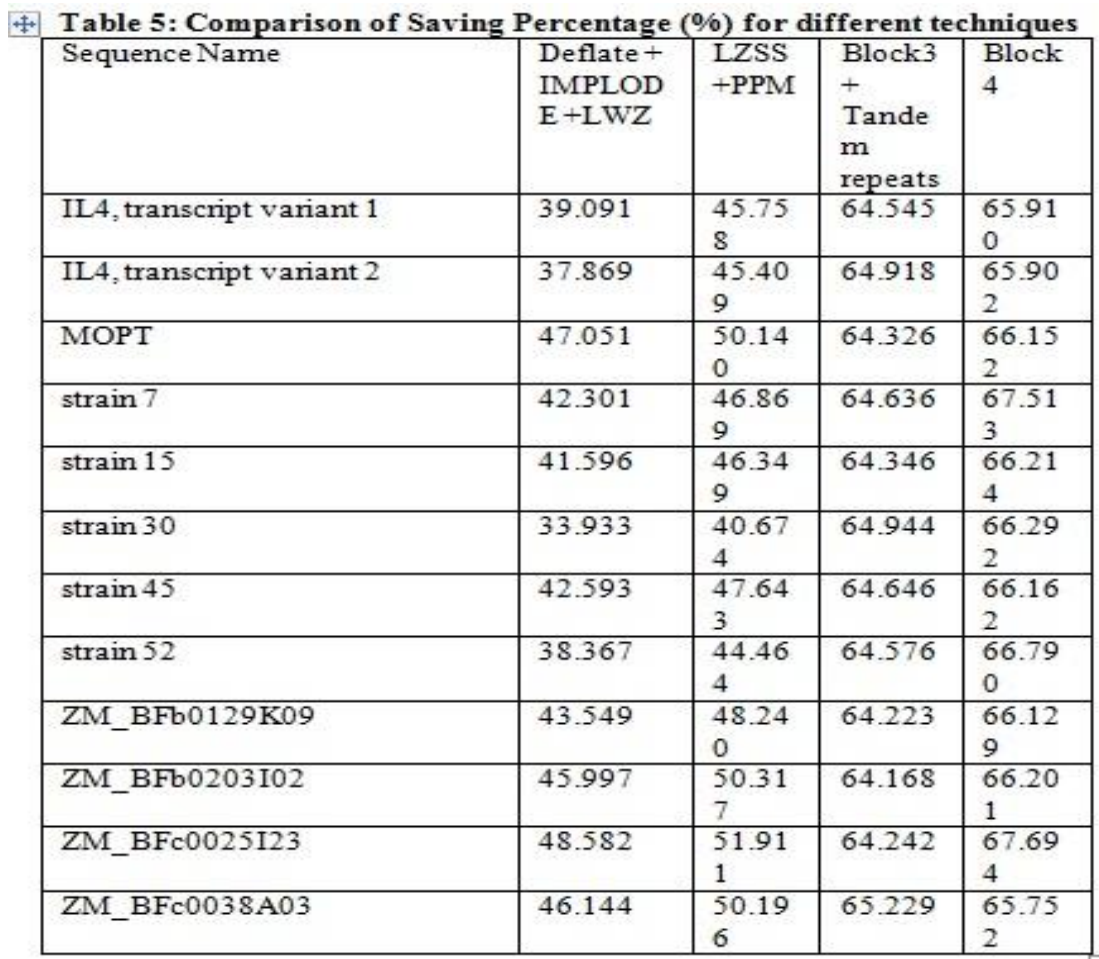

The graphical representation of table 3 is shown in fig-1

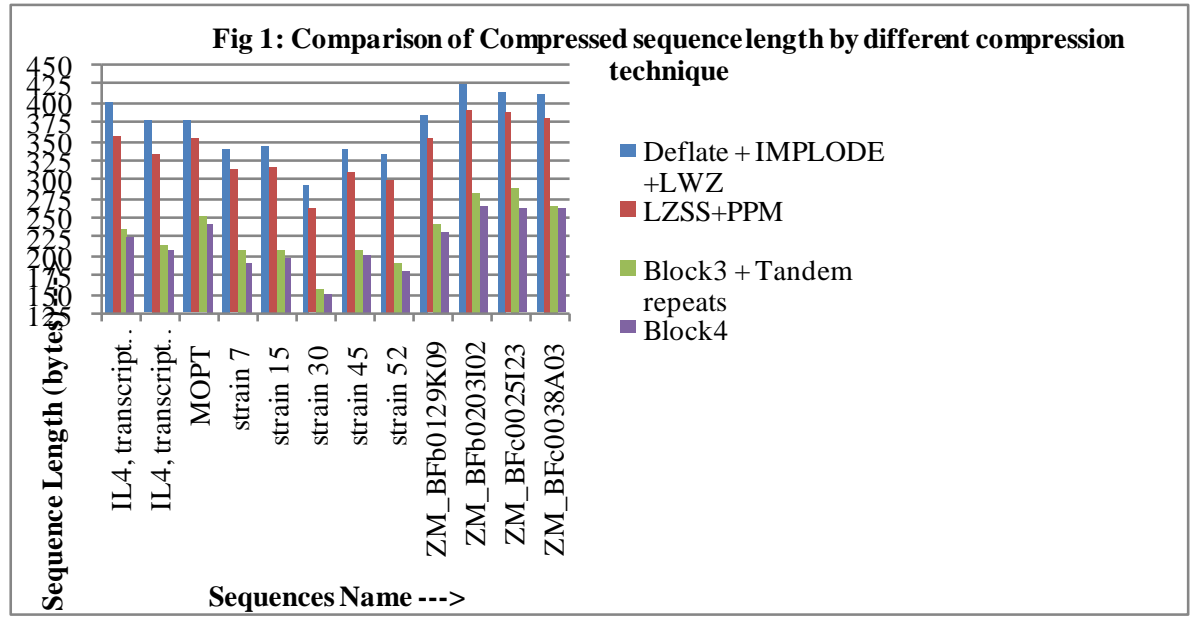

The graphical representation of table 4 is shown in fig-2

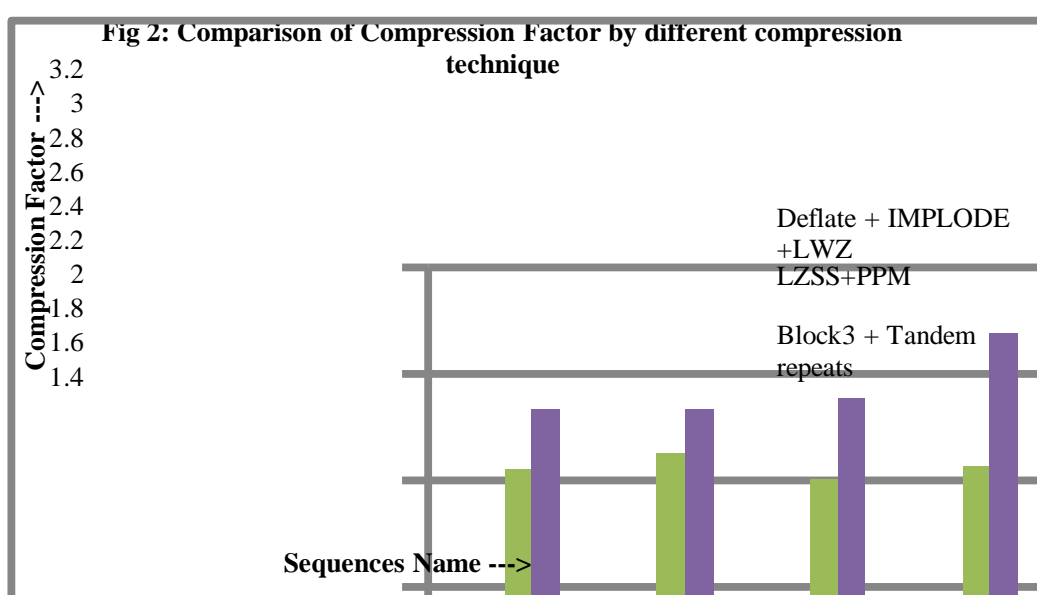


The graphical representation of table 5 is shown in fig-3

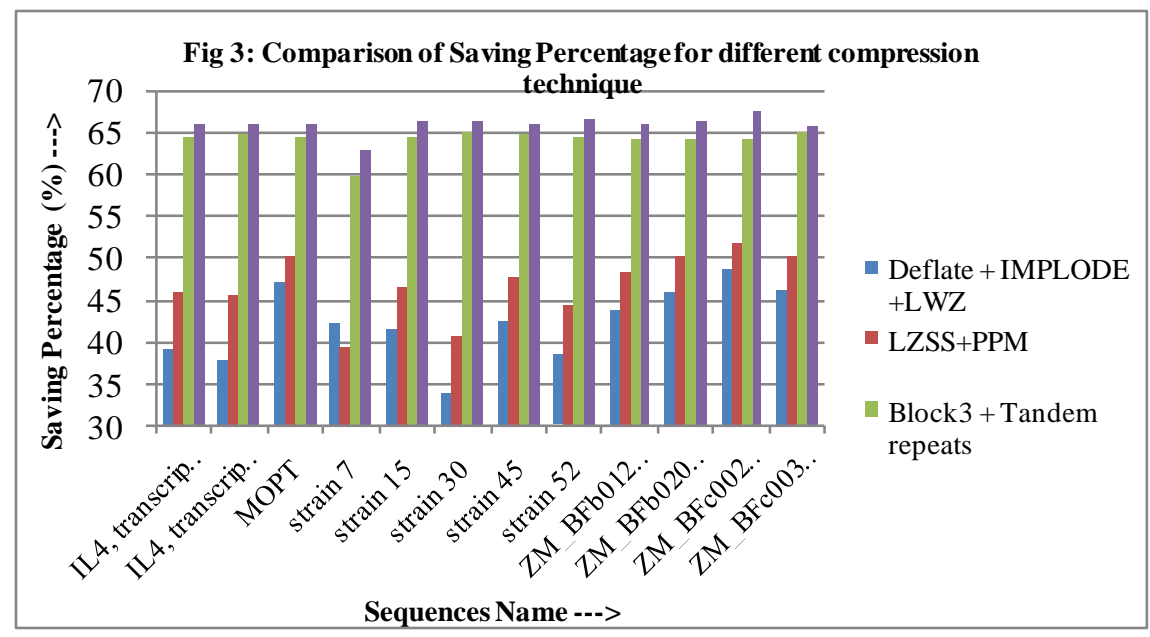

\section{Conclusion and Discussion}

In this article, we have presented two new compression algorithms that are based on LUT of block size three with tandem repeats and LUT of block size four.

There are various most common compression algorithms like LZSS, WinZIP use deflate, deflate64, IMPLODE, dynamic LWZ, WinRAR use LZSS and PPM etc but they are not giving good result for the compression of biological sequences as the result shown above that our propose compression algorithms gives better result.

The propose algorithms have high compression factor and giving good saving percentage to other exiting Biological Sequence Compression. This technique also requires less memory and less coding effort compare to the other algorithms.

The proposed techniques compress Biological sequences taking block size of three, four and using tandem repeat in the sequence line. So it will be easier to make sequence alignment and sequence analysis between compressed sequences.

\section{References}

[1] Genbank size from: ftp://ftp.ncbi.nih.gov/genbank/gbrel.txt

[2] Hyoung Do Kim and Ju-Han Kim , "DNA Data Compression Based on the Whole Genome Sequence", Journal of Convergence Information Technology Vol. 4, No. 3, September 2009.

[3] Pothuraju Rajarajeswari, Allam Apparao, "DNABIT Compress - Genome compression algorithm" Biomedical Informatics, volume 5, Issue 8, pp. 350-360, 2011.

[4 ] P.Raja Rajeswari and Dr. Allam AppaRao, "GENBIT COMPRESS TOOL (GBC): A java-based tool to compress DNA sequences and compute compression ratio (bits/base) of genomes", IJCSIT, Vol. 2, No. 3, pp. 181-191, June 2010.

[5] P.Raja Rajeswari, Dr. Allam AppaRao and Dr. R. Kiran Kumar, "HUFFBIT COMPRESS - Algorithm to compress DNA sequences using extended binary trees", JTAIT, pp. 101-106, 2010.

[6] Heba Afify, Muhammad Islam and Manal Abdel Wahed, "DNA lossless differential compression algorithm based on similarity of genomic sequence database", IJCSIT, Vol. 3, No 4, August 2011.

[7] R.K.Bharti, "A Biological sequence compression Based on Approximate repeat Using Variable length LUT", International Journal of Advances in Science and Technology, Vol. 3, No.3,PP:71 -75, 2011.

[8] R.K. Bharti, Prof. R.K. Singh, “A Biological Sequence Compression based on Look up Table (LUT) using Complementary Palindrome of Fixed Size”, ICJA (0975-8887), Volume 35- No.11, December 2011.

[9] R.K.Bharti, "Biological sequence Compression Based on Cross chromosomal properties using variable length LUT", CSC Journal, Vol. 4 Issue 6, PP: 217-223. , 2011.

[10] Sequences are taken from: http://www.ncbi.nlm.nih.gov/.

[11] Suman Chakraborty, Sudipta Roy, Prof. Samir K. Bandyopadhyay, "Image Steganography Using DNA Sequence and Sudoku Solution Matrix", International Journal of Advanced Research in Computer Science and Software Engineering(IJARCSSE), Volume 2, Issue 2, February 2012. 